\title{
The role of family history and its influence on the onset time in female pattern hair loss
}

\author{
Adriana Łukasik, Karolina Kozicka, Agata Kłosowicz, Andrzej Jaworek, Anna Wojas-Pelc
}

Department of Dermatology, Jagiellonian University Medical College, Krakow, Poland

Adv Dermatol Allergol 2021; XXXVIII (5): 815-818

DOI: https://doi.org/10.5114/ada.2020.100745

\begin{abstract}
Introduction: Female pattern hair loss (FPHL) is one of the most common causes of hair loss in women. Genetics plays an important role in the development of the disease, but the etiopathogenesis and the inheritance pattern in women remain unexplained.

Aim: To determine the extent to which FPHL is of genetic origin in women from the Polish population and whether a positive family history is a risk factor for its earlier onset.

Material and methods: Family histories of 111 unrelated female patients with FPHL and 129 female patients without hair thinning were analysed. FPHL was diagnosed based on a detailed medical history, the clinical picture and trichoscopic features.

Results: A positive family history was noted in 69 (62.2\%) patients with FPHL. In 32 (28.8\%) patients from that group, more than one person in the family suffered from hair loss, whereas in the healthy group, the same was true of only 4 patients $(3.1 \%)(p<0.0001)$. A positive family history on the mother's side proved statistically significant for FPHL patients. In the case of 20 (18\%), hair loss had been identified in their grandparents, while the healthy group had a negative history in that respect. A positive family history of hair loss in grandparents was three times more frequent in the group of patients with the disease onset before 40 .

Conclusions: A positive history on the mother's side may be of great significance for FPHL development. Hair loss in more than one family member and in one's grandparents may also indicate a higher risk of disease development.
\end{abstract}

Key words: family history, female pattern hair loss.

\section{Introduction}

Androgenic alopecia is the most common cause of hair loss both in women and in men. It is a chronic disease and it progresses with time. The onset time and intensity vary depending on the geographical location, but the intensity always tends to increase with age [1-4]. The highest prevalence is noted in the Caucasian population (29-56\% of patients older than 70 ) [2-4], while the lowest one is observed in the Asian population (12-25\% of patients over 70$)[1,5,6]$. The prevalence of hair loss in the family is also significantly higher in the white population [7]. In a study conducted by Wang et al. in the Chinese population, family history was positive for $19.2 \%$ of female patients [5], while in a study carried out by Paik et al. in Korea, that value reached $45.2 \%$ [1].

It is presently believed that the mode of inheritance is polygenic and multifactorial, but it has not been thoroughly examined in women yet.
To arrive at a correct diagnosis and exclude other potential causes of hair loss, a thorough medical history should be taken, including the duration of symptoms, intensity of hair loss, currently taken medications and the applied treatments. A trichoscopic examination is presently the gold standard in diagnostics.

\section{Aim}

The aim of the study was to evaluate the inheritance pattern of female pattern hair loss (FPHL) in women from the Polish population and potential influence of a positive family history for its earlier onset.

\section{Material and methods}

We qualified 111 unrelated female patients for the study. They were treated in the Department of Dermatology in Krakow in 2018-2019, and diagnosed with female

Address for correspondence: Adriana Łukasik, Department of Dermatology, Jagiellonian University Medical College, 50 Kopernika St, 31-501 Krakow, Poland, phone: +48 505156 827, e-mail: adrianalukasik2@gmail.com Received: 24.07.2020, accepted: 18.08.2020. 
pattern hair loss based on the clinical picture and the trichoscopic examination. Trichoscopy was performed with a handheld dermoscope and videodermatoscope. The diagnosis of FPHL was based on the presence of 2 major criteria or 1 major and 2 minor criteria. Major criteria included an increased number of yellow dots (more than 4 yellow dots in the frontal area in 4 fields of vision at 70-fold magnification) and thin hairs, decreased average hair thickness in the frontal area and more than $10 \%$ of thin hairs $(<0.03 \mathrm{~mm})$ in the frontal area. Minor criteria included an increased frontal area to occiput ratio of single-hair units (>2:1), vellus hairs $(>1.5: 1)$ and follicles with perifollicular discoloration (> $3: 1)$ [8].

The control group included 129 women without hair thinning and with a negative history of pathological hair loss. Some patients in the control group were treated in the Department of Dermatology for other skin diseases which had no influence on hair loss. A detailed family history concerning hair loss, taking into account the patient's grandparents, parents, siblings and offspring, was collected in both groups. The onset time of the first symptoms (hair loss or hair thinning) was also noted, as stated by the patients.

\section{Statistical analysis}

The data are presented as numbers of cases and proportions expressed in percentages. We compared the studied group with the control group with respect

Table 1. Number of patients in the different age groups

\begin{tabular}{lcc}
\hline Age of patients & \multicolumn{1}{c}{ Number of FPHL patients $(n=111)$} \\
\cline { 2 - 3 } & $n$ & $\%$ \\
\hline $17-29$ & 8 & 7.20 \\
\hline $30-39$ & 27 & 24.32 \\
\hline $40-49$ & 28 & 25.22 \\
\hline $50-59$ & 21 & 18.91 \\
\hline $60-69$ & 17 & 15.31 \\
\hline $70-79$ & 9 & 8.10 \\
\hline $80-89$ & 1 & 0.90 \\
\hline
\end{tabular}

to the family history, using Pearson's $\chi^{2}$ test, with Yates correction if the expected number of observations was less than five. A two-sided $p$-value of $<0.05$ was considered significant. The Statistica 10 software (StatSoft Inc., Tulsa, Oklahoma) was used for the calculations.

\section{Results}

The average age of patients was similar in both groups (47 in the control group, and 48.4 in the studied group).

\section{The age distribution of FPHL patients in the study}

The youngest FPHL patient was 17 years old, while the oldest one was 86. Patients aged 30-49 constituted the biggest group among the FPHL patients (49.5\%). Table 1 shows the number of patients in the different age groups.

\section{The role of family history}

The family history of hair thinning in the conducted study was positive for 69 (62.2\%) FPHL patients, and for $36(27.9 \%)$ patients from the control group. A positive family history concerning more than one person in the family, taking into account three generations back, was established for 32 (28.8\%) patients in the studied group, and only 4 (3.1\%) patients in the healthy group $(p<0.0001)$ (Table 2 and Figure 1$)$.

Patients in the control group did not mention hair loss in their grandparents. However, the family history was positive for the grandparents of 20 patients from the FPHL group (Figure 2).

\section{Family history and the age of onset}

The earliest onset reported by a patient in our study was at the age of 12. In that case, the family history was positive for the mother, the father and all four grandparents, and hair loss intensity was assessed as very high. A positive family history of hair loss in grandparents was three times more frequent among patients with the onset before the age of 40 (15 vs. 5 patients).

However, no statistically significant influence of hair loss in the family history on an earlier onset of FPHL was

Table 2. A comparison of positive family histories with respect to selected family members for FPHL patients and women from the healthy group

\begin{tabular}{lccc}
\hline Family history & $\begin{array}{c}\text { FPHL group }=111 \\
n(\%)\end{array}$ & $\begin{array}{c}\text { Control group }=129 \\
n(\%)\end{array}$ & $\begin{array}{c}P \text {-value } \\
\text { Mother }\end{array}$ \\
\hline Father & $32(28.8)$ & $5(3.8)$ & 0.005 \\
\hline Mother and maternal family & $34(30.6)$ & $6(15.5)$ & $<001$ \\
\hline Father and paternal family & $36(32.4)$ & $21(16.3)$ & 0.046 \\
\hline Parent & $31(27.9)$ & $25(19.4)$ & $<0.0001$ \\
\hline
\end{tabular}


observed for the other patients, especially those aged 17-39 $(p=0.6257)$ (Table 3).

\section{Discussion}

Hair loss in the family is of great significance for FPHL development.

Our study revealed hair loss in one parent in the case of $53 \%$ of patients, which is comparable with the results of a study involving 30 female patients, conducted by Tandon et al. in India [9], where hair loss in first-degree relatives was noted in $46 \%$ of patients, and a study by Paik et al. [1], where a positive family history concerned approximately 40-54\% of female patients, especially for an early-onset FPHL. The present study reveals no relationship between a positive history and an earlier onset of the disease, except for 1 case in which a very early onset (the twelfth year of life) correlated with a strongly positive family history.

In a study carried out by Wei Siah et al., which involved a group of 210 female patients with FPHL, the family history was positive for $85 \%$ of the patients ( $51 \%$ on the father's side, $20 \%$ on the mother's side, and $24 \%$ on both sides). The history was negative in $14 \%$ of patients [10]. Smith and Wells also observed a higher prevalence of hair loss in firstdegree male relatives of women with FPHL than in those of healthy women [11].

In our study, the family history was positive for $62 \%$ of patients with FPHL The prevalence of hair loss in male- and female-line relatives was comparable, with a slight predominance of a positive history on the father's side (34 vs. 32). A positive family history on the father's side also prevailed in the control group.

Taking into account the hair loss prevalence on the father's side ( $n=20$ in the control group, and $n=34$ in the studied group) and on the mother's side ( $n=5$ in the control group, and $n=32$ in the studied group), a statistically significant difference is observed only for hair loss on the mother's side.

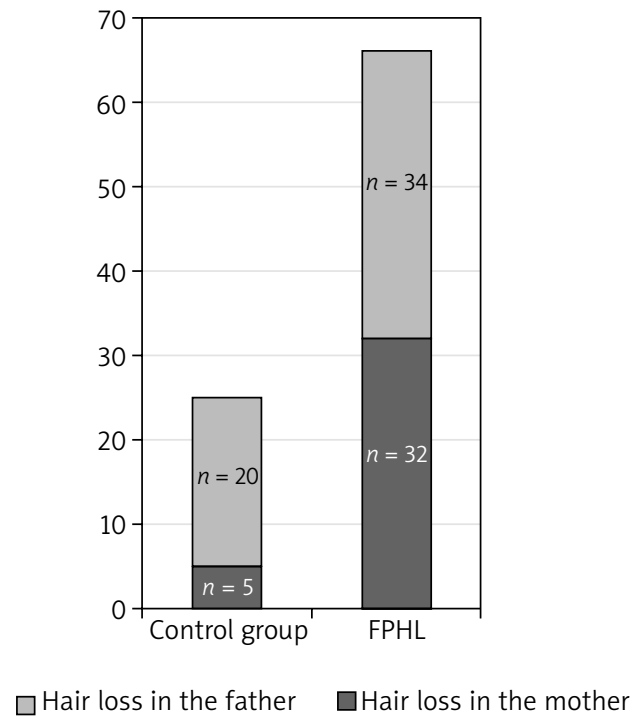

Figure 1. A comparison of hair loss prevalence on the mother's and father's sides in the FPHL group and the control group

Table 3. The onset time of hair loss symptoms in correlation with the family history

\begin{tabular}{lccc}
\hline Onset & \multicolumn{3}{c}{ Family history } \\
\cline { 2 - 4 } & Number & Positive & Negative \\
\hline $12-25$ & $23(20.7 \%)$ & $17(73.9 \%)$ & $6(26.1 \%)$ \\
\hline $26-35$ & $18(16.2 \%)$ & $9(50.0 \%)$ & $9(50.0 \%)$ \\
\hline $36-45$ & $23(20.7 \%)$ & $16(69.6 \%)$ & $7(30.4 \%)$ \\
\hline $46-55$ & $24(21.6 \%)$ & $14(58.3 \%)$ & $10(41.7 \%)$ \\
\hline $56-65$ & $14(12.6 \%)$ & $8(57.1 \%)$ & $6(42.9 \%)$ \\
\hline $66-80$ & $9(8.1 \%)$ & $5(55.6 \%)$ & $4(44.4 \%)$ \\
\hline
\end{tabular}

A similar hair loss prevalence on the father's side in the FPHL group and the control group can be related with the fact that androgenic alopecia is more frequent in

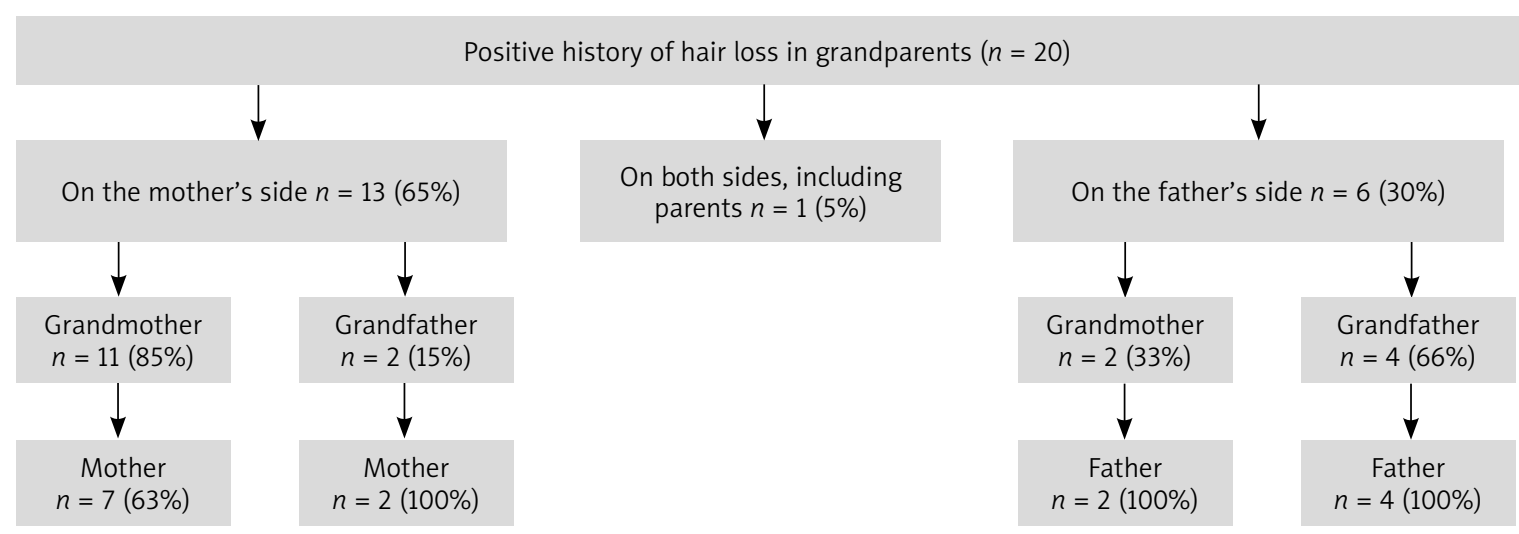

Figure 2. The percentage distribution depending on a positive history on the mother's and father's sides 
men, ranging from $50 \%$ among 50 -year-old men to nearly $80 \%$ over the age of 70 in the Caucasian population [3].

This observation may indicate a greater significance of the family history on the mother's side in the case of female hair loss. The higher hair loss prevalence in maternal grandmothers may also support this.

Similar results were presented by Salman et al. [7], who noted a significantly higher frequency of a positive history in the mothers and sisters of women with FPHL in comparison with healthy women; however, no such relationship was observed for second-degree relatives.

Moreover, the study did not observe a relationship between the FPHL onset time in women with a positive history on the mother's side, but it did note such a relationship in men with a positive history on the father's side.

Contrary to the yet unclear significance of family history in women, a positive family history is undoubtedly a risk factor for androgenic alopecia in men. A study conducted in a male population by Chumlea et al. [12] established the greatest significance of a positive history in the maternal grandfather and the father. Similar results were obtained by Nargis et al. [13], where a positive family history was found in $68 \%$ of patients, including 44 (62.8\%) with a positive family history in paternal relatives, and only $8.6 \%$ with a positive family history in maternal relatives.

This suggests a much greater influence of familial hair loss in male-line relatives on men with AGA.

\section{Conclusions}

A positive family history of hair loss concerning more than one person in the studied group confirms the significant role of a positive family history in disease development. A positive family history in over $50 \%$ of the female patients may indicate the autosomal dominant inheritance pattern of the trait.

The hair loss prevalence in the studied group was similar on the mother's and father's sides, but this might be related to the higher prevalence of androgenic alopecia in men than in women worldwide. The frequent positive family history with respect to the grandparents of women with FPHL highlights the role and the necessity of taking a detailed family history, when assessing the disease development risk at the initial stages of hair loss as well as the necessity of conducting further genetic research.

Strengths: To our best knowledge, this is the first study concerning the genetic origin of female pattern hair loss in women in Poland.

Weaknesses: Lack of ability to verify the type of hair loss/type of alopecia in the family. Lack of knowledge of the entire family structure.

\section{Conflict of interest}

The authors declare no conflict of interest.

\section{References}

1. Paik J, Yoon J, Sim W, et al. The prevalence and types of androgenetic alopecia in Korean men and women. Br I Dermatol 2001; 145: 95-9.

2. Birch MP, Messenger JF, Messenger AG. Hair density, hair diameter and the prevalence of female pattern hair loss. Br J Dermatol 2001; 144: 297-304.

3. Gan DC, Sinclair RD. Prevalence of male and female hair loss in Maryborough. J Investig Dermatol Symp Proc 2005; 10: 184-9.

4. Norwood OT. Incidence of female androgenic alopecia (female pattern alopecia). Dermatol Surg 2001; 27: 53-4.

5. Wang T, Zhou C, Shen Y, et al. Prevalence of androgenetic alopecia in China: a community-based study in six cities. Br J Dermatol 2010; 162: 843-7.

6. Lin-Hui S, Li-Sheng C, Hsiu-Hsi C. Factors associated with female pattern hair loss and its prevalence in Taiwanese women: a community-based survey. J Am Acad Dermatol 2013; 69: e69-77.

7. Salman K, Altunay I, Kucukunal L, et al. Frequency, severity and related factors of androgenetic alopecia in dermatology outpatient clinic: hospital-based cross-sectional study in Turkey. An Bras Dermatol 2017; 92: 35-40.

8. Brzezińska-Wcisło L, Rakowska A, Rudnicka L, et al. Androgenetic alopecia. Diagnostic and therapeutic recommendations of the Polish Dermatological Society. Dermatol Rev 2018, 105, 1-18.

9. Tandon S, Arora P, Gautam RK, et al. Correlation between clinical features, biochemical parameters, and histopathological findings in women with patterned baldness: a study from North India. J Cutan Aesthet Surg 2019; 12: 42-8.

10. Wei Siah T, Muir-Green L, Shapiro J. Female pattern hair loss: a retrospective study in a tertiary referral center. Int J Trichology 2016; 8: 57-61.

11. Smith M, Wells R. Male-type alopecia, alopecia areata, and normal hair in women: family histories. Arch Dermatol 1964; 89: 95-8.

12. Chumlea WC, Rhodes T, Girman CJ, et al. Family history and risk of hair loss. Dermatology 2004; 209: 33-9.

13. Nargis T, Vishal B, Pinto $M$, et al. Early onset androgenetic alopecia in men and associated risk factors: a hospital based study. Int J Res in Dermatol 2017; 3: 267-71. 